\title{
Concerning the Madrid Lecture: The Equivocal Character of Pavlov's Reflexological Objectivism and its Influence on the Distorted Concept of the Physiology-Psychology Relationship
}

\author{
Juan Bautista Fuentes \\ Complutense University of Madrid
}

\begin{abstract}
The aim of this study is to bring to light and make clear the characteristic equivoque Pavlov made when he conceived the neurophysiological circuit involved in conditioned behavior as if this circuit contained or reduced, in its own terms, such behavior. I also wish to point out how this interpretation implies a distorted concept of the relationships between neurophysiology and behavior. As an alternative, it is proposed that behavior, which is always operant behavior, is not at all reducible to its neurophysiological ingredients, but instead acts like an arrow-head for adaptation that, in turn, confers a subordinate function to the neurophysiological ingredients involved. To make this evident, behavior is considered an operant organic activity that takes place in an environment of "remote co-present" texture, and different from its neurophysiological ingredients, which take place within the context of spatial contiguity relationships.

Keywords: physiology, psychology, reflexological objectivism
\end{abstract}

\begin{abstract}
Este trabajo pretende desvelar y deshacer el equívoco característico en el que incurrió Pavlov al entender el circuito neurofisiológico involucrado en la conducta condicionada por él descubierto como si este circuito contuviese o se redujese, en sus propios términos, a dicha conducta, y señalar de qué modo esta interpretación implica una concepción distorsionada de las relaciones entre neurofisiología y conducta. Alternativamente, se propone que la conducta, que además es siempre conducta operante, no es en modo alguno reductible a sus condiciones o ingredientes neurofisiológicos, sino que funciona siempre como la punta de lanza de la adaptación en cuyo seno tienen sentido funcional subordinado sus propias condiciones o ingredientes neurofisiológicos por ella involucrados. Para demostrar esto se propone entender la conducta como actividad orgánica operante dada en un ambiente de textura "co-presente a distancia", a diferencia de sus ingredientes neurofisiológicos que se darían en el contexto de las relaciones por contigüidad espacial. Palabras clave: fisiología, psicología, objetivismo refelxológico
\end{abstract}

Correspondence concerning this article should be addressed to Juan Bautista Fuentes, Sección Departamental de Psicología Básica II, Facultad de Filosofía, Ciudad Universitaria, 28040 - Madrid (Spain). E-mail: jbfuent@ filos.ucm.es 


\section{General Presentation of the Aim and the Issue Addressed in this Study}

The aim of this study is to show how, in the course of his discovery and interpretation of conditioned reflexes, Pavlov characteristically incurred in the equivoque of considering the neurophysiological circuit involved in the conditioned behavior that he discovered not only as a neurophysiological process, but also-and here is the root of the equivoque-as if this process contained, absorbed, or reduced such behavioral activity. As we shall see, initially, this allowed Pavlov to advocate an alleged reductionist explanation, insofar as it was neurophysiological, of a supposed mental activity that he always regarded from a mentalist and representational-dualist viewpoint. At a later stage in his thinking, Pavlov finally modulated and radicalized this claim by an explicit assumption of some kind of reductionist fusion of the neurophysiological process and the supposed mental activity.

As I will attempt to show in this paper, the error incurred by the above-mentioned equivoque lies basically in the unawareness of the fact that behavioral activity is not at all reduced to the neurophysiological process always involved in such activity, but instead that this process should only be considered as the necessary involved neurophysiological ingredient of behavior. Therefore, behavior, insofar as it is not reduced to this neurophysiological ingredient, should always be maintained, both conceptually and experimentally, within its own behavioral scale so that researchers can conjecture and eventually identify and record the neurophysiological ingredient involved. Thus, they can eventually understand the functional meaning of this neurophysiological ingredient of behavior as immersed in the organism's integral adaptation within its environment. The arrow-head of this adaptation, impossible to eliminate from an adaptive viewpoint and physiologically irreducible, is, precisely, behavior.

In effect, only if these issues are understood in this way is it possible not to distort the meaning of the integral psychophysiological functional unity of organic adaptation and, hence, the very meaning of biological research. And this distortion occurs, whether, like Pavlov, we consider neurophysiological functioning to contain or reduce, in its own terms, behavioral activity, or whether we believe that a behavioral approach to behavior can be meaningful (beyond a mere animal behaviortraining technique). Although the behavioral approach is always technically possible, it could ignore the crucial biological issue of the behavior-physiology relationship. This course of action has been more characteristic of the psychological guild, whose paradigm was habitually established by behaviorisms.

Therefore, the aim of this study is to analyze and clarify, with logical precision, this equivocal Pavlovian concept of the behavior-physiology relationship. I will also try to critically detect the scope and influence of this ambiguity in the very relations between what are, in fact, two moments, the physiological and the psychological, within the field of biological research.
The Presence of Psychological Issues in the Field of Encephalic Physiology of Integral and Unrestrained Organisms

In the first place, Pavlov's discovery of the conditioned reflexes must be considered an example of the general process by which modern experimental physiology, once it ceased to be spinal physiology and necessarily became encephalic physiology — or, as Pavlov stated, a physiology that must focus on the whole and unrestrained organism-, must deal with psychological issues. This means exploring the issues revealing the behavioral dimension of an organism's integral adaptation as the necessary content, formally internal, to its field. Such a process began to take place around the second half of the XIX century, in sensory and motor physiology laboratories. It would also inevitably appear in the Pavlovian laboratory at the end of the XIX and the beginning of the $\mathrm{XX}$ centuries, in the field of the physiology of the secretory digestive glandular reflexes.

Spinal physiology, by means of its spinal preparations, had managed to obtain, in an experimentally pure way, the system of reflexes whose nervous connection centers could only be spinal or of the brainstem. This is the organism's last (or first, depending on one's point of view) defense system, comprised of a type of reactions that, in a normal state, can simultaneously coincide with the animal's alert, conscious, or voluntary activity, but in a more or less automatic way and, to this extent, with some functional autonomy with regard to conscious activity. Therefore, to obtain this automatic functional autonomy in an experimentally pure state, spinal preparations were necessary. This implied physiologists' awareness of the fact that, in an organism's normal state, these reactions could be integrated within the encephalic neurophysiological activity involved in the organism's conscious or voluntary activity. The way in which spinal physiology designated and conceptualized these spinal reflex reactions as $u n$-conscious or in-voluntary reactions is very significant. Using a privative/negative prefix with respect to the organism's conscious or voluntary activity, the rest of the neurophysiological activity of the integral organism was acknowledged as always involved in the organism's conscious or voluntary activity. Spinal physiology always acknowledged the fact that the integral organism's encephalic neurophysiological functioning (including spinal functioning, inasmuch as it could be integrated within encephalic functioning) was, in fact, involved in the organism's obviously conscious and voluntary activity.

Despite the above-mentioned need for the physiology of spinal preparations, this physiology would have a very limited field of action and it would therefore be necessary to extend physiology's sphere to a morpho-physiologically integral organism. The issue is, when physiology becomes physiology of a morpho-physiologically "integral" organism, the organism must be "behaviorally unrestrained," that is, in a condition to display its behavioral and/or cognitive 
activity (there is not the slightest opposition between these aspects, inasmuch as they both occur together). Therefore, physiological research will now be forced to incorporate this behavioral activity into its field, to the extent that the physiological functions to be studied are not functionally detachable from the activity or behavioral moment in the organism's global or integral adaptation to its environment.

All of this had to happen and did, in fact, happen in the context of sensory and motor physiology, around the second half of the XIX century. It was impossible to study the physiology of each sensory organ without also taking into account its corresponding perceptual activity because the physiological functioning of these organs is only activated when immersed in such perceptual activity. Therefore, this perceptual activity had to be considered the very condition of the pursuit of physiological research. Similarly, it was impossible to study the physiology of motor activity without also taking behavior into account. Whereas the physiological functioning of the musculature was observed to be a morpho-physiological condition of support and channeling of this behavior, such physiological functioning would only be activated and operate when immersed in cognitively oriented behavior. Behavior (that is, the "voluntary" or "conscious" movements) had to be taken into account as the very condition of pursuit of physiological research.

Encephalic physiology of integral and unrestrained organisms could no longer disregard the psychological or behavioral dimension of the organism. Therefore, it must address the crucial issue of the type of relationships and differences between both moments, the (neuro)physiological and the behavioral, within the organism's global adaptation to its environment, in other words, the psycho-physiological issue. Even when the most characteristic ideology of the guild was prevalent, namely, the ideology that tends to support a reductive explanation of this behavioral moment in neurophysiological terms, there was constant controversy regarding other possible opinions which always tried to reduce this behavioral dimension neurophysiologically.

Pavlov's discovery of the "conditioned reflexes" is no doubt another case of this general process commented upon and one that characterizes the emergence of encephalic physiology around the second half of the XX century.

\section{Pavlov's Experimental Discovery of and Approach to the "Conditioned Reflexes"}

In the course of his research of such an apparently "humble" process as the physiological digestive functions of experimental organisms, Pavlov verified over and over that these digestions could not be separated from the very diverse environmental situations perceived by the organism, that somehow concurred with the moment of "contact" of the nutritive substance with the stomach walls responsible for the digestive secretions. The secretions clearly took place, not only when the substances acted on contact with the digestive walls, but also when the organism perceived the diverse environmental situations that had accompanied this "contact."

From then on, Pavlov had to aim his research at establishing an experimental design capable of exercising systematic control over the process by which his organisms performed the "psychological digestions" that he encountered time and again in his laboratory. As formulated in Pavlov's basic canonical terms, the conditioned learning process by which a perceived environmental situation, that at first only elicits a cognitive orientation reaction, can, due to its repeated temporal association with a stimulus that elicits an "unconditioned" ("universal" or "absolute") reflex reaction, as a result of its conditioning or reinforcement by the unconditioned stimulus, eventually elicit a reflex reaction that is similar (at least qualitatively) to the unconditioned reaction. The situation thus becomes a conditioned discriminative situation (which is still called "stimulus") that subsequently acts as an anticipatory signal for the unconditioned stimuli on which it depends.

Thus, Pavlov bequeathed to subsequent psychological research the technique, the method, or the procedure of experimental achievement and control of conditioned reflex responses. However, Pavlov was a physiologist, which means he worked in a field that could not overlook the biological functional unity of the psychological and physiological dimensions of the organic adaptation, no matter which solution was subsequently chosen about the relation between both moments of this living functional unity. Therefore, Pavlov could not limit himself to the experimental control and reconstruction of the psychological process of conditioning reflex responses, but instead became interested in the neurophysiological functioning involved in this psychological process, as well as the crucial issue of the relationship between both moments of the organism's living functional unity. This is the fundamental "classic" importance or scope of Pavlov's work, which I feel should not be ignored or disregarded. I refer to his tenacious refusal, typical of the biological perspective, to dissociate or break the living functional unity of the psychological and physiological moments of the organic adaptation. His ulterior solution to the problem of the relationships between both moments of the organic adaptation could — and, I believe, should—be radically and critically revised, as I will attempt to show below.

First, note that the proposals made insistently and systematically by Pavlov throughout his work about the neurophysiological processes involved in conditioned behavior always consisted of conjectures or hypotheses corelated or co-responding to the behavioral situations he had discovered and controlled. So, only on the basis of these effective experimentally controlled behavioral situations could these neurophysiological conjectures be meaningfully conjectured or proposed. These neurophysiological 
conjectures were meant to co-respond to the behavioral situations that had been discovered and addressed. Moreover, the format of the conceptual content of these conjectures clearly corresponds, in the form of neurophysiological vocabulary, to the format of the basic behavioral situations discovered or addressed by Pavlov. Therefore, the mutually correlative processes of cortical "excitation" and "inhibition" correspond with the behavioral processes correlative to "reinforcement" and "extinction" (of a conditioned stimulus-CS-by its corresponding unconditioned stimulus-US). Similarly, the cortical processes of "irradiation" and "concentration," either of excitation or of inhibition, correspond, respectively, with "generalization" and "discrimination" (of the CS), which may be reinforced or extinguished (by the presence or absence of the US). Likewise, the "opening" of a "new nervous connection tract"-in contrast to the "conduction tracts" already morpho-physiologically presented by the unconditioned reflexes-between cortical zones and the spinal centers again corresponds with the behavioral situation of temporal association of the CS (discriminative and/or generalized) and the US, an association in which reinforcement (and therefore conditioning) takes place. Pavlov often mentioned the establishment of "temporal nervous connections" that correspond with the temporal behavioral associations between the CS and the US obtained experimentally in the laboratory. With regard to this subject, readers can consult, among others, the global and late presentation of Pavlov's work on conditioned reflexes in his 1934 article entitled "The Conditioned Reflex", written for The Great Medical Encyclopedia.

On the other hand, it is a well known fact that Pavlov himself barely had the opportunity to independently identify and record the neurophysiological processes he conjectured, but rather it was his school, especially following the works of Ukhtomsky, that could begin to obtain the pertinent physiological registers.

However, I do not mean to suggest, as would someone with a behaviorist attitude, that such neurophysiological conjectures could be irrelevant by their mere redundancy. On the contrary, I suggest that these conjectures should be made in order to identify and record, in a positive and independent way, their contents. In doing so, we will not lose sight of the living functional unity of the organic adaptation and therefore, be forced to deal with the inexorable biological issue which is crucial to the psychophysiological relationship. In this sense, Pavlov himself cautioned that restricting oneself to merely obtaining and controlling conditioned behavior was nothing more than simple training, if such work were not complemented and completed with knowledge about the neurophysiological processes involved in the behavior. In effect, at the Madrid Lecture, which I will subsequently mention, Pavlov stated: "Evidently, a great number of surprising facts when training [italics added] animals belong to the same category [italics added] as some of our experiments" (Pavlov, 1903/1982, p. 117, from the Spanish edition of 1982).

In effect, there is an aspect of this physiological part of biological work which must be emphasized, as Pavlov himself always did. It is necessary to take into account that the neurophysiological circuit (cortical-sub-encephalic) involved in behavioral activity should, no doubt, be closed in neurophysiological terms, because otherwise, behavioral activity would be deprived of its indispensable conditions of morpho(neuro)physiological support and channeling. In this case, we would be entering the sphere of parapsychology or telepathy. Hence, as a matter of principles, independent of the initial conjectural nature of Pavlov's neurophysiological proposals and the specific degree to which his school or general neurophysiology may have been able to identify and record the neurophysiological processes involved in behavior, the tenacious Pavlovian refusal to dismiss those processes clearly means that the neurophysiological circuit involved in the behavior must necessarily and unavoidably be closed, in terms of neurophysiology, so as not to lose sight of the living functional unity of the organic adaptation.

Now then, the point is that it is one thing to need to consider the neurophysiologically closed nature of the neurophysiological circuit involved in behavior, and another thing to incur in the equivocal concept, as did Pavlov, of con-founding, or considering this need to be the same as the presumed reductionist capacity of this closed neurophysiological circuit to explain behavior. It is as if the organism's integral adaptation to its environment could be confined and limited to this neurophysiological circuit, abstraction made of behavior, when it could be that the neurophysiological circuit of such support and channeling would only be functionally activated and closed (for each behavioral cycle or pattern) via the very mediation of behavior, and insofar as behavior is not reduced to its conditions of morpho(neuro)physiological support and channeling. A concept operation is, in effect, "equivocal" when "one thing is considered to be another," thus judging in an equivocal or erroneous way. And what I wish to state here is that Pavlov, and many others before and after him, erroneously treated the necessary neurophysiological closed nature of the neurophysiological circuit involved in behavior as if said circuit reduced behavior. It may actually be that the circuit is only activated and closed when immersed in maintained behavioral activity, and precisely insofar as such activity cannot be reduced to the circuit.

By means of this equivocal concept, Pavlov helped to promote a distorted concept which hindered the relationship between behavior and physiology. Let us see how Pavlov incurred in this equivocal concept, and subsequently we shall discuss or criticize his own discussion of the issue and, thus, try to clarifty this equivoque. 
The Pavlovian Discussion of the NeurophysiologyBehavior Relationship

I will try to limit this presentation of the Pavlovian discussion of the behavior-physiology relationship to his classic work "Experimental Psychology and Psychopathology in Animals"(Pavlov, 1903/1982), the lecture Pavlov presented one hundred years ago at the International Congress of Medicine in Madrid. During this lecture, Pavlov presented his discoveries on conditioned reflexes for the first time. It is extremely significant that it was at this first presentation that our physiologist had to deal with the treatment and discussion of the psycho-physiological issue, as an issue raised by the same discoveries he was presenting for the first time.

Pavlov began his speech with the statement: "You will hear how a physiologist was forced to go [italics added] from purely physiological problems to the domain of phenomena usually termed psychological." According to Pavlov, this was because these "manifestations of a psychological order" which "I encountered during a long period of research ... on the normal activity of the digestive glands .... contribute considerably and permanently to the good progress of the studies considered" (Ibid., p. 109, from the Spanish edition). As we can see, Pavlov began his speech from the perspective of a biologist who realized that, from within the physiological field, the organism's psychological activity must be considered a functionally internal moment of its global adaptation to its environment, inasmuch as this psychological activity contributes considerably and permanently to the sound progress of events. However, one notes a certain cautious tone as Pavlov spoke of his own definite conclusions. Instead of mentioning just "psychological phenomena," he spoke about phenomena "normally considered" psychological. This precaution no doubt has to do with his final conclusion, that is, to reach a reductive methodological-objective explanation, inasmuch as physiological, of these psychological phenomena in neurophysiological terms. Pavlov forewarns us about this at the beginning when he notes that "despite its suddenness, this transition happened in a totally natural way and, more importantly, without changes in the methodological principles" (Ibid., p. 109, from the Spanish edition). However, despite the reductionist aim insinuated at the beginning of his speech, Pavlov would be forced to address the problem of the relationship between the physiological and the psychological, of the organism's integral adaptive functioning. And he would have to deal with this issue in terms that I consider indispensable, even though in the end, he would finish by trivializing the complexity of the issue.

Pavlov began addressing the problem in terms of the differences and the relationships between the "distance" discriminative-conditioned situations (the so-called conditioned "stimuli") and the organism, and the obligatory "action by contact" on the organism by the unconditioned physiological stimuli. In effect, Pavlov began by acknowledging that the basic difference that must be perceived between a "purely" physiological reflex (unconditioned) and a "psychological reflex" (conditioned) lies in the fact that "in the physiological form of the experiment, the substance is placed in direct contact with the organism, whereas in psychological experiments, it acts at a distance" [italics added] (Ibid., p. 113, from the Spanish edition). Thanks to this "direct contact," in the case of the unconditioned reflex, the physiological response to the eliciting stimulus can be understood. "In the physiological case, -according to Pavlov-the activity of the salivary glands is linked to the properties of the substance upon which the saliva will act. Saliva moistens and lubricates the matter that must be swallowed and neutralizes the effects of chemically active substances, and this is precisely the function of the special stimulators of the specific mouth surfaces" (Ibid., p. 113, from the Spanish edition). However, in the "psychological experiments," the organism also reacts by salivating, but now in environmental situations that Pavlov himself had to acknowledge were "at a distance" from the organism. Therefore, in this case, the response (salivation) is elicited by the properties of these distant objects or situations, properties that Pavlov had to acknowledge in perceptual terms:

In the psychological experiments the animal is stimulated by properties of the external object that are not essential for the action of the salivary glands, or even by properties that are totally accidental or lacking in importance: the visual, auditory, or even purely olfactory properties of our objects .... For example, the plate on which food is presented, the furniture on which the plate is placed, the room, the person who usually presents the food, and the noises made by the person, even if he cannot be seen at that time .... Presenting our hand, which smells of powdered meat, to the animal will be enough to elicit salivation. Also, the sight of the food at a large distance ... is capable of provoking the action of the salivary glands .... Thus, in the psychological experiments, the connection of the objects that stimulate the salivary glands becomes more and more distant and delicate [italics added] .... The importance of these remote signs [italics added] (signals) of the objects can easily be seen in the reaction of the animal's movements, when it searches for objects, avoids enemies, etc. (Ibid., p. 114, from the Spanish edition)

What can be said about this distinction, established by Pavlov himself, between "action by contact" and "remote connection" to characterize, respectively, the functional correlation between stimulus and response in the "purely physiological" case (unconditioned reflexes) and the functional correlation between perceived environmental situation and the reaction in the "psychological case" (conditioned reflex)? Certainly, as Pavlovian research and writings advanced, we note a decrease in the frequency with which he refers to 
unconditioned and conditioned reflexes, respectively, in terms of reflexes elicited by "contact" and reflexes elicited by "remote" situations. Nevertheless, this type of reference does not completely disappear, but instead it re-emerges precisely on the occasions when Pavlov has to re-address, explicitly and formally, as in the Madrid Lecture, the problem of the relationship between behavior and physiology. As an example, we can look at his work "Physiology and Psychology in the Study of Animals' Higher Nervous Activity," a report presented at the Philosophical Society of Petrograd in 1916, and published in the Psychiatric Gazette in 1917 (see pp. 287-300, from the Spanish 1982 edition).

My proposal is that such a distinction is absolutely indispensable, crucial and decisive. This is why Pavlov could not cease to consider it even though he never really displayed sufficient critical awareness of how much was implied in this distinction. Hence, Pavlov naively believed that he could solve the problem posed by simply observing that the physical distance between the situation that is the object of perception, and the organism's surface, should in turn be filled with a physical-energetic stimulation set that would act "by contact" with other receptor organic surfaces. It was as though by this observation the problem addressed would be solved, a problem that is in fact the very problem of perception. Thus, for example, in the work we are commenting upon, Pavlov concluded by saying, "But if we consider it in more detail, we will see that there is no essential difference between these experiments - in reference to the psychological experimentsand the purely physiological ones. The difference is that, in the psychological experiments, the substances act upon other body surfaces-the nose, the eyes, the ears-via the surrounding medium [italics added] (air, ether, etc.) in which both the organism and the excitatory substances are immersed" (see Pavlov, 1903/1982, p. 113, from the Spanish 1982 edition).

Now then, by such an observation, all Pavlov has done is to restrict himself to noting the necessary physicalenergetic stimulation process that must operate, via "contact" relations, between the remote physical source that is the object of perception and some perceptive organ so that there could be perception. Observe that, via this reference to the physical-energetic process of stimulation by contact, what Pavlov is looking for is to be sure of the closure, in terms of relations by contact, of the physiological-environmental circuit that must be present in perception and, together with perception, the process of behavioral conditioning. But at the same time, Pavlov converts the equivocal concept, consisting of con-founding this physiological-environmental circuit closed by contact, into the very process of perception and of behavioral conditioning. Pavlov's entire argument strategy is reduced to noting the neurophysiologicalenvironmental circuit closed by contact, which must be present in perception and in behavioral conditioning, and supposing, implicitly and erroneously, that the very process of behavioral conditioning is reduced to this circuit.
What I therefore propose is that there is no doubt that this neurophysiological circuit closed by contact comprises the necessary condition or material ingredient of the perceptive and behavioral conditioning process, but that the perception and the behavioral conditioning it implies, cannot be resolved or reduced to its material condition. That is, it is necessary to clarify how perception and behavior, considered formally and in act, cannot be resolved or reduced to what comprises their necessary conditions or material ingredients. I refer to the neurophysiological-environmental circuit involved in this behavior, a circuit whose activation and operation only make functional sense when immersed in perceptive and behavioral functioning, inasmuch as the latter cannot be resolved or reduced to the circuit.

However, before going into this crucial issue, I would like to mention two modulations that, at different times during the evolution of Pavlov's thinking, his reductionist equivoque adopted.

In the first moment, this reductionism adopted a methodological-objective nature, which meant accepting an explicit mentalist concept, of a dualist-representational type, of psychological life. Pavlov accepted a concept of psychological life as a presumed mental-representational content, enclosed within the mind of each organism, and to which other organisms (and man) would have no kind of direct access. Hence, the possible conjectures about that supposed mental life made by comparison with one's own mental life lacked a minimum inter-subjective validity in order to guarantee the methodological-objective reliability that can only be obtained by knowledge of the physiologicalenvironmental circuit closed by contact. What Pavlov proposed is a kind of indirect access, methodologically reliable due to its exclusively physiological content, to a supposed mental life, understood in the most naïve mentalist terms, those of a dualist-representational nature. The following words from the Madrid Lecture, seem to be sufficiently representative:

What should the physiologist do with these data-in reference to "psychological" data? .... Should we penetrate an animal's inner state and represent in our own way its sensations, feelings, and desires to arrive at a comprehension of these new facts? .... To achieve more knowledge of the facts, can we compare an animal's inner state [italics added], even though it may be as highly developed as the dog, with our own? .... In our psychological experiments with the salivary glands, at first we tried to explain in detail our results, giving free rein to our imaginations about the animal's possible subjective state. We achieved nothing but sterile discussions and some isolated and incompatible personal opinions. We had no choice [italics added] but to carry on with our experiments in a purely objective [italics added] field, proposing the urgent and important task of resisting our natural tendency to refer our own state to the animal's reactive mechanism. (Ibid., p. 112, from the Spanish edition) 
With time, however, perhaps under the influence of the official Soviet scholastic metaphysics of dialectic materialism - with which Pavlov lived pragmatically-, he seems to have accepted the ontological thesis that assumes some kind of (reductionist) merging of mental life, which is still conceived of in mentalist terms of a dualistrepresentational nature, and "higher nervous activity." Thus, neurophysiological explanations would no longer be an indirect, although methodologically reliable and objective way of access to mental life, but they would somehow imply direct access to mental life, inasmuch as it merges with higher nervous activity. For example, in his prologue to IvanovSmolenski's book, Fundamental Problems of Pathophysiology of Higher Nervous Activity, in 1933, we can read:

We see the rough draft, a natural and inevitable approach, a merging of [italics added] psychological with [italics added] physiological, of [italics added] the subjective with [italics added] the objective. The problem that has accompanied human thinking will find a 'concrete' solution. The sublime mission of science in the near future will consist of contributing the maximum to this merging. (see Pavlov, "On the Possibility of the Fusion of the Subjective and the Objective,” p. 333, from the Spanish 1982 edition)

Also see Pavlov's work of 1934, "The Conditioned Reflex," where he states:

The temporal nervous relation is a universal physiological phenomenon in the animal world and in human life. It is also a psychological phenomenon that psychologists call an association. Why must we distinguish, separate, what the physiologist calls a temporal relation and the psychologist an association? We are in the presence of a complete fusion, of a total mutual absorption, of an absolute identity [italics added]. (p. 184, from the Spanish 1982 edition)

One notes how the postulated merging is, in any case, reductionist in a physiological sense, because it is a merging of the psychological or subjective with the physiological or objective, and not the opposite.

The problem is that both versions of the Pavlovian reductionist equivoque depend on the same type of incapacity on which the equivoque is based, that is, the incapacity to discern how behavior is not formally reducible to its necessary neurophysiological ingredients or material conditions. Thus, only if we are capable of discerning this crucial issue will we then be able to clarify both the dualistrepresentational concept of psychological life as a supposedly enclosed or internal mental content, as well as the naïve and confused idea of the merging of this assumed mental content with physiological activity. And only then will we perceive what seems so difficult for all the "objectivists" (who, in turn, are always implicitly or explicitly "mentalists"): That psychological life consists of behavior and only of behavior.
This is so because behavior cannot be not resolved or explained physiologically, that is, by its necessary supporting and channeling ingredients or material conditions. We shall now, finally and directly, deal with this issue.

\section{Criticism of the Pavlovian Discussion of the Neurophysiology-Behavior Relationship}

As previously mentioned, Pavlov restricted himself to observing the physical-energetic stimulation process, which, operating by contact, should doubtless "fill in" the physical distance between the remote source of stimulation and some perceptive organ. However, in this way the issue of what perception really is has not been addressed or resolved, but rather only its necessary (material) conditions, and therein lies Pavlov's equivoque.

In order to distinguish this difference, and not commit the same equivoque, I propose that it was necessary for Pavlov to have had more knowledge in certain areas that he seems to have ignored. I am referring to the discovery of the "perceptive constancies." A discovery that, I believe, allows one to capture the "secret" of psychological life, that is, the cognitive or perceptual link of organisms with their environment.

What this discovery experimentally reveals - in reference to the most schematic nucleus-is that the qualities of subjectively perceived objects (for example, the observed size or form of an object) correlate highly or predominantly, although never totally, with the physical properties (subject to measurement by the experimenter) of the remote or distant objects that are actually perceived (for example, the physical size or form measured). They are therefore relatively independent of the variability of the proximal stimulation that comes from the remote objects (for example, the physical size or form of the retinal image).

This means that distal physical sources should never be reduced to or coincide with the proximal physical stimulation, because the notion that perception is perception of proximal stimulation is functionally meaningless, as its meaning is to be found in the idea that perception must be perception of what is remote, only inasmuch as it remains physically remote.

I propose a more general and comprehensive explanation of the meaning of the perceptive constancies - that is, the fact that perception is only functionally meaningful when it is of remote objects, inasmuch as they remain remote. Therefore, note the crucial difference between the morphologies and trophic functions of autotrophic and heterotrophic organisms and the necessary relationship between these differences and the functional meaning of the cognitive link with the environment.

Autotrophic organisms, which are capable of carrying out their trophic functions via photosynthesis of inorganic substances that operate by physical contact with their body surfaces, don't need either the morphology or the function 
of local movement, nor do they need digestive systems to carry out their trophic functions.

On the other hand, heterotrophic organisms must ingest organic substances (vegetable, animal, or both), that, in general, are not in contact with their bodies, but instead are remote from their bodies. Therefore, they must develop two types of morphologies and functions: not only digestive systems to ingest and digest such substances, and to excrete the non-nutritive residuals, but also motor organs for local movement in the environment, so they can cover the physical distances that separate them from these living nutritive substances, and seize them in order to ingest them-all the more so when these substances are, in turn, mobile, that is, animal or heterotrophic organisms.

But then, I suggest that the only way to understand this "presence" of the remote, inasmuch as it remains remote, in a non-metaphysical manner (in this context: in a non dualist-representational manner), is by means of the idea of "remote co-presence" (of what remains physically remote): "remote co-presence," in fact, between the various sectors, regions, or strata of the physical environment which are physically distant from each other, and always with regard to the movements of the organism that, in turn, must adopt the texture of these "remote co-presences."

The idea of "remote co-presence," proposed herein, to characterize the "formal texture of the environment" where behavior occurs, is certainly not unheard of in psychological literature. A very relevant classical discussion of the adequate level of analysis of behavior in terms of "remote relations" between the "distal focuses" between which the "behavioral achievement" occurs, was developed by Brunswik in many parts of his work (for example, in Brunswik, 1938, and in Tolman and Brunswik, 1935), and very particularly in his later work of 1952, The Conceptual Framework of Psychology. A critical discussion of this author's proposal to adjust the "distal" nature of behavioral achievements within the positivist (or behaviorist) methodological physicalist framework can be found in the "Introduction" to my translation into Spanish and critical edition of Brunswik's work (1952) (see Fuentes, 1989).

Note that the idea of "remote co-presence" does not mean "remote action," as Pavlov has sometimes naively stated. In effect, at the Madrid Lecture, Pavlov considered the possibility of some "remote action" between the CS and the organism. He later discarded this possibility noting that the energetic stimulation set, proceeding the distant physical source subsequently operates "by contact" on the organism. "At first sight-says Pavlov-whereas in the physiological form, the substance enters into direct contact with the organism, in the psychological form, it acts at a distance (italics added)" (p. 113 of the Spanish 1982 edition). However, one should not even consider the possibility of "remote distance" because all action should continue operating by "spatial contiguity." It is a case of "remote co-presence," which does not challenge spatial contiguity, but which is not reduced to this contiguity in its formal texture. In effect, if we do not wish to enter the realm of parapsychology or telepathy, "action" should continue to be used instead of "spatial contiguity." But, for the purpose of cognitive functions, "remote co-presence" means the "evacuation" of such spatial contiguity, that is, of the "solutions of continuity" via spatial contiguity, which we must assume go on occurring at all times. Hence, the always "copresent" "texture" of the perceived objects cannot be resolved or formally reduced to any of the possible solutions of spatialcontiguous continuity, in whose scope the necessarily copresent texture of all perception is just diluted or submerged.

The notion of "remote co-presence" allows one to sidestep the (metaphysical) muddles that are typical of representational dualism; that is, both the concept of knowledge as an presumed inner, or enclosed, mental representation of an also supposed physical reality juxtaposed with its presumed representation, as well as the concept of behavior as an activity, the organism's body movements, imprisoned within the supposed physical world juxtaposed with its also supposed inner mental representation. And this allows one to understand how behavior and knowledge operate together, immersed in the co-present environmental texture.

It is very important to note that perception is inseparable from movement (from the motor activity of local motion) as much as movement is inseparable from perception. Perception, or the co-present texture of the environment (texture that also includes certain parts of the organism's moving body) only has functional meaning if we take into account those movements that might be cognitively oriented within this texture. Therefore, we must consider "behavior" to be only and exactly the organic movements performed within this co-present texture of the environment (and therefore cognitively oriented). This texture, in turn, only makes sense if we take these movements into account. An effective cognitive "configuration" will only be achieved in the course of movement, which is now behavioral as its performance is cognitively oriented.

We can then understand that the behavioral "functional arcs" are behavioral just because they occur always within this co-present texture of the environment. Any behavioral "functional arc" (pattern, cycle) is made up of some sequence of transformations of cognitive configurations, effected by the organism's own behavioral movements, which are differentially selected from among possible alternative transformations, as a function of some hedonic experience (appetitive or aversive) that so functionally cancels out the functional cycle. Therefore, both the effectively cognitive nature of every cognitive configuration of this functional arc as well as the various alternative links between these configurations are only behaviorally possible because they occur within an environment of co-present texture. At the level of spatial contiguity (which must be considered as operating uninterruptedly), the likelihood of reaching such cognitive configurations and unfolding their various mutually alternative links is diluted or submerged. This is only possible in the co-present texture of environment. 
Behavioral activity, taken as organic mobility occurring within the co-present (or cognitive) texture of environment, cannot be resolved or formally reduced, in its own adaptive functionality, to its materially essential ingredients. Rather, such activity acts like an "adaptive arrow-head," functionally ineradicable, inasmuch as irreducible to its morpho-physicalecological spatial contiguous conditions. By means of this "adaptive arrow-head" (a) the organism accommodates, actively and selectively modifying the physical environment with which its morphophysiology is "compared" by spatial contiguity. Therefore, (b) it is only within this maintained behavioral activity that the functioning of the morpho-physioecological spatial contiguous circuit involved in behavior can make any functional sense. Intuitively stated, an organism, hypothetically equipped with the same neurophysiological activity as that involved in a behavioral organism, but deprived of its effective behavioral activity, would be cognitively blind and/or behaviorally incapable of performing the integrity of the organic adaptive functions that it can only perform as a result of its behavioral activity. And so, such hypothetical neurophysiological functioning would be, eo ipso, functionally inert or superfluous. This is what we could call the paradox of the "Cartesian Automaton." Individuals who believe they can reduce behavior to neurophysiology incur in this paradox frequently and naively. This means, therefore, that, despite possible (reductionist) appearances, the morpho-physio-ecological, contiguousspatial conditions of support, and channeling of behavior are functionally dependent on their behavioral context (and not vice versa).

This is only clear when we can let go of the singular block or conceptual masking that the "experimental Pavlovian artifact" inflicts on behavioral activity. I propose that the Pavlovian technique of conditioning is not really conceptually neutral, but instead is related to some deep conceptual defect. I refer to the assumption that it is possible to experimentally obtain these reflex responses making a formal abstraction of instrumental or operant behavior, without whose active mediation, it is totally impossible to acquire such conditioned reflex response. Some link between a situation and some other reinforcer could be obtained experimentally, so that the situation would elicit the same reflex response as did the "unconditioned" stimulus, only if, in the organism's past experiences there was some operant behavior, by whose mediation, the organism actively related the discriminative situation with the reinforcing situation, and by whose mediation the "Pavlovian effect" was obtained. Any organic movement that occurs in a co-present environment and by whose performance or active mediation some cognitive configuration is achieved and a reinforcing situation is obtained, is "operant." All behavior is "operant," so that even perception should be considered operant behavior, insofar as any operant behavior can only act through the continued exercise of perception. Thus, the socalled "Pavlovian effect" should be considered a reaction that must nevertheless have been acquired or conditioned in the course of some operant conditioning activity. Therefore, its functional behavioral meaning is to act like an anticipatory emotional reaction of the reinforcing goal, obtained by the operant activity, and this is why it works. It is a conditioned "activation syndrome," which, according to specific thresholds in each case, can either be a facilitator or an inhibitor of the operant task in question.

We must adopt the compulsory viewpoint of the AngloAmerican functionalist "law of effect" tradition. For example, in his classical work "The Reflex Arc Concept in Psychology," Dewey (1896) had already established the basis for a monofactorial and operant learning concept like the one advocated in this article (see Fuentes \& Quiroga, 2001). Only when we adopt such a functionalist viewpoint can we give up the conceptual error that is typical of the Pavlovian tradition. According to Pavlov, his "conditioned responses" are presumably "behavioral units," supposedly having functional behavioral meaning "by themselves," that is, isolated from the operant course within which they are acquired and have functional behavioral meaning. No doubt, the effect of such an abstraction is to give the impression that operant behavior is susceptible to being formally "factorized," or "de-composed" and "re-composed," in terms of chains of stimuli and peripheral reactions or in terms of the necessarily spatial-contiguous nexuses on the scale where these S-R chains occur. However, the only effective behavior, that is, operant behavior, inasmuch as behavioral activity on the scale of its co-present texture, is no longer formally solvable in terms of these spatial contiguous nexuses that characterize its necessary peripheral physiological ingredients.

To consider the so-called conditioned "stimuli," and even the reinforcers, as "stimuli," and to suppose that temporal contingency associations between them can be understood from a "metric" concept of time, is just a resource intended to offer an ostensible verbal appearance of "objectivity" or of "scientificness." The so-called (in the Pavlovian and behaviorist traditions) conditioned "stimulus" is not a stimulus at all, because the only stimuli that exist are proximal energetic excitations that influence some receptor tissue by spatial contiguity, and it is functionally meaningless for these proximal stimulations to be the object of perception. Perception can only be perception of remote objects as long as they remain remote, that is, perception of the distal physical sources of proximal stimulation, so that these sources, inasmuch as they are perceived, must possess some co-present texture that makes their nature of perceived "objects" possible. This cognitive characteristic would be entirely diluted or submerged in any of the nexuses of spatial contiguity that fill in the physical distance between the identified distal sources and proximal stimulation. In fact, these configurational situations work and are experimentally treated (both in the Pavlovian and the behaviorist traditions) like "discriminative" and/or "generalized" situations (which are those that are differentially reinforced). These concepts themselves imply the presence 
of some perceived configurational situation, and they are entirely invalidated as soon as we enter the realm of effective relations of spatial contiguity. Therefore, to refer to "discriminative stimulus" is totally meaningless. Similarly, this also occurs with the so-called reinforcing "stimuli," because their reinforcing function within each behavioral cycle lies precisely in their hedonic experience (appetitive or aversive). This experience can only acquire its experiential character as long as it already possesses some co-present texture, no matter how small. That is, in order for the organism to obtain and maintain this experiential character, shifting, no matter how small, of the effective proximal stimulations involved is unavoidably required, and this shifting is performed by organic movements. Thus, (in terms of perceptive constancies), such shifting can open up a minimum path of variability of proximal stimulation so that, with some independence of this variability, no matter how little, these experiences can be achieved, maintained, or transformed, as minimal perceptive constancies.

It would also be naïve to think that the temporal contingency associations (or the "accidental and delicate" temporal connections, according to Pavlov) of the discriminative situations and the hedonic experiences can take place within a formally metric time, instead of occurring, as they do, within a constantly behavioral time, that is, within a period of time that is formally "patterned" by "sequences" or "transitions" of operant "transformation" between various configurational discriminative situations that eventually lead to some hedonic experiential situation. When we enter the realm of "metric" time, that is, when we factorize behavioral time in terms of "consecutive successions of movement between the parts of a place that are formally connected by spatial contiguity relations" (on some spatial-contiguous scale, for example, the one formed by a mechanical clock), then we are setting up the metrictemporal ingredients that act as the material condition of the effective behavioral time. However, this behavioral time, formally and in act, is not reduced to these ingredients. Instead, it is only within this behavioral time that the specific type of "behavioral variability," which is characteristic of such "contingent interdependencies" or "accidental and delicate" connections, can have any meaning. As a result of such connections, learning takes place; the organism never ceases to cope with and modify its environment by means of the "flexible" or "ductile" alternative character of such interdependencies or connections. Such flexibility, ductility, or malleability cannot take place within metric time, which is always of a spatial-contiguous nature.

Now then, only when we understand the co-present texture on whose scale behavior moves formally (given that this activity is not reducible to its spatial contiguous morphophysio-ecological ingredients), are we then in a position to deal with this issue with some conceptual precision, so we can relinquish the equivocal concept always implied in the reductionist physiologist assumption.
Proposal to Invert the Standard Concept of the Relations between Behavior and Physiology

And so, I maintain that it is necessary to proceed to "invert" the established or "standard" (physiological reductionist) concept of the mutual conceptual modeling relationship between behavior and physiology. This inversion was the idea behind the "old" classic Gestalt hypothesis on functional isomorphism between the behavioral field and central neurophysiological functioning. The most important part of this hypothesis was that it inverted the concept of established (representational-dualist) "common sense" that always tends to consider neurophysiological functioning a "substrate," or "base," and therefore, a conceptual "model" of psychological life.

Only when we have realized that behavior cannot be formally reduced to the necessary relations of spatial contiguity at the scale of which should exist the neurophysiological nexuses of the "nervous activity," can we begin to realize that the nervous activity involved in behavior can adopt functional forms of organization that somehow must be similar to those of the same behavior in action, notwithstanding the obligatory spatial-contiguous structural (histological) channeling. This similarity was pointed out in the "classic" Gestalt notion of a topological (not topographical) and functional isomorphism of central neurological activity with regard to behavioral activity. In regard to this, it is extremely important to remember, for example, the discussion by Koffka (1935) about this issue in the second chapter of his classic book Principles of Gestalt Psychology.

This topological and functional isomorphism can and should be understood in terms of a plastic and zonal functioning. Brunswik called the principle of that functioning a "vicarious micro-mediation" (Brunswik, 1952). "Vicarious micro-mediation" means the permanently plastic and zonal neurological functioning where the various "structural parts" always work "jointly" to some extent, and therefore, always have some room for mutual inter-substitutability or equifunctionality with regard to their functional goal. This functional goal is behavior itself; which, given its co-present texture, is always occurring so that genuine discrimination, no matter how small, will always have some degree of generalization and, subsequently, its various components can be considered functionally intersubstitutable and discriminative.

In sum, the neurological performance implicated in behavior, notwithstanding its necessary spatial-contiguous structural channeling, must adopt functional organizational forms in accordance with the functional organization form of co-present behavior. Only in this way can we understand (a) that behavior is this "moment," physiologically irreducible inasmuch as adaptively not eliminable, of the integral organic adaptation, and (b) starting at this moment, and only at this moment, inasmuch as it is maintained, can the necessary 
conjecture and eventual identification of the neurophysiological performance involved in behavior make any sense. The functional organization forms of this performance are functionally dependent or subordinate and, therefore, "in accordance with" behavior.

Thus, we can finally observe not only Pavlov's tenacious biological refusal to break up the functional-psychophysiological unit of living beings, but also the fact that the actual conceptual format of his neurological conjectures (cortical "inhibition" and "excitation;" their "irradiation" and "concentration;" the opening of "new encephalic-spinal connection tracts") was functionally isomorphic with the conceptual format of the effective behavioral situations he discovered ("reinforcement" and "extinction:" "generalization" and "discrimination" that were reinforced or extinguished, and the formation of "accidental or delicate" "new temporal connections"). It is very ironic that the man who dedicated a good part of his efforts to criticize the Gestalt school from his reductionist physiologist viewpoint should, by his choice of format in physiological concepts, confirm the daring and subtle Gestalt concept of the relations between physiology and behavior.

Lastly, Pavlov's equivoque and the reductionist physiological pretense that it facilitates, are closely linked to Pavlov's aim of understanding the content of the "higher nervous activity" involved in behavior in "reflexological" terms. In other words, in terms that are conceptually based on the spinal nervous arcs that are characteristic of "unconditioned" reactions, despite the attainment novelty and structural and functional complexity that these higher "cortical reflexes" are known to possess. The "reflexological" assumption was assumed at first independently, and almost simultaneously by Pavlov and Bechterev, in both cases as a result of the influence of their common master, Sechenov. But in the following text, we can observe the extent to which the idea that cortical nervous activity is "reflex," was a façade, because of the incapacity to understand how behavior, always operant, is not reducible to its neurological conditions. In this sense, subsequent attempts by psychology officially connected to the Soviet scholasticism of dialectic materialism to discard the idea of the physiologically reflex nature of cortical activity-for example, in the case of Kornilov, when proposing "reactology" instead of "reflexology"-still retain the worst feature of the reflexological assumption: The concept of organic activity that, instead of being permanently directed by the arrow-head of operant behavior and not reducible to its neurological ingredients, is a mere "reactive" physiological system considered, at best, to be "merged" with a presumed mental activity conceived in a representational-dualist way. Official Soviet psychology never managed to free itself from the reflexological prejudice, insofar as this prejudice converged with the mentalist and representational-dualist concept, proposed by Lenin in Materialism and Empiric Criticism. This concept considered knowledge to be a "reflex" or "representation" of an outer reality. Representational-dualist mentalism was thus the effective nexus between the official Soviet ideology and the Russian "reflexological" physiologists' tradition: hence, the stage for the confused idea of "fusion" between subjective activity, presumably mental, and objective brain activity, presumably "reflexological," was set.

However, only by means of an appropriate concept of behavior as operant organic activity with co-present texture, can we appreciate that the only level of organic animal activity that can be understood in terms of "reflex nervous arcs," is the level made up of spinal reflexes. And this is so only to the extent that these reflexes can be considered in their relative functional autonomy with regard to their possible integration in the encephalic nervous activity involved in behavior. Moreover, not even the concepts of "reflex" or "reflex arc," because of their Cartesian origin and the "optical-mechanical" model that this implies, are appropriate to conceptualize these effector reactions whose nervous centers can be considered exclusively spinal. This optical-mechanical model, insofar as it is adopted from a ray of light shining on a mirror and the corresponding light "reflected" from the mirror, contradicts the actual functional complexity of the simplest spinal reaction. Whereas this model does not expect the reflected ray to be able to influence and modify its emitting source, the simplest organic reaction is always functionally a "circular reaction" ("feedback," as it was subsequently called), in which the stimulation must be actively affected and modified by the reaction so that the reaction will be functionally fulfilled, and in which there is always room for "selective accommodation" of the response with regard to the stimulus. This is so even within the exclusively spatial contiguous relations that are not yet behavioral. The optical-mechanical "reflex" model does not reflect any of this, rather it betrays it. In fact, if the tradition of spinal physiology has maintained the concept of "reflex," despite its insufficiency, it has only been to refer to the somewhat "automatic" nature and the relative "functional autonomy" (with regard to the "higher" nervous activity involved in behavior) of spinal reactions. This functional autonomy should be conceived according to the criterion proposed herein, while acknowledging that the functional complexity of these reactions and their characteristic nervous spinal arc is not reflected upon in the optical-mechanical concept of reflex. In this sense, it is interesting to note that even Pavlov, perhaps unknowingly, followed the same conceptual privative-negative strategy seen in classic spinal physiology when conceptualizing spinal reflexes as "in-voluntary" or "un-conscious" activity, because he conceived spinal reflexes as "un-conditioned" reflexes, that is, reflexes that are not yet psychologically conditioned but that are susceptible to being conditioned.

Notwithstanding this conceptual "optical-mechanical" deficiency left over from the physiological tradition, Pavlov's intention of conceptualizing the "higher" nervous activity on the model of nervous spinal "reflex" activity, constitutes 
a new and different error added to the first one. In the Pavlovian conditioned response, the sole "reflex" is that its effector topography (glandular, visceral, or motor) is similar to the topography of "reflex" spinal reactions. So, although its effector topography is still similar to that of "reflex" spinal reactions, the neurophysiological functioning involved, like the operant behavior where it occurs, will be isomorphically in accordane with that behavior. That is, it should operate according to some pattern of corticalencephalic "vicarious micro-mediation" in accordance with the behavioral "vicarious macro-mediation" in which it is involved, a pattern that cannot be conceptually modeled by "reflex" spinal arc functioning.

The "reflexological" concept of the "higher nervous activity" involved in behavior is then a mere facade to accomplish or complete the conceptual error that considers that the conditioning of "Pavlovian" reactions is possible independently of the mediation of operant behavior. If this error creates the appearance that effective operant behavior is susceptible to being factorized or resolved in terms of peripheral physiological S-R chains whose topography is similar to that of reflex spinal reactions, this appearance is reinforced and completed if one assumes that these chains can be effectively integrated by higher nervous activity of a supposedly reflex nature.

And so, the mirage of the Pavlovian "reflexological objectivism," according to which behavior is reduced to and contained in terms of "higher nervous activity," is definitely achieved. This mirage allowed Pavlov to believe that the neurophysiology of the supposed "cortical reflexes" would offer a scientific-or objective inasmuch as reflexologicalexplanation of psychological life that Pavlov, in turn, always understood from a mentalist viewpoint of a representationaldualist nature.

However, in this work, I have made an effort to show that only when one has an appropriate concept of behavior as an operant organic activity within a co-present textureand therefore, neurophysiologically irreducible and adaptively inevitable, does the very action of its contiguous-spatial neurophysiological ingredients make subordinate functional sense. Only then can one: (a) discard any mentalist concept of psychological life of a dualist-representational nature and substitute it with an effectively behavioral concept, and therefore (b) dismantle the mirage that implies that psychological life can be reductively explained by a presumably reflexological physiology whose conceptual format depends on the equivocal concept that assumes that behavior-that is, the only effective psychological lifeis contained and limited within this supposedly reflexological physiology.

\section{References}

Brunswik, E. (1938). Psychology as a science of objective relations. Philosophy of Science, 4, 227-260.

Brunswik, E. (1952). The conceptual framework of psychology. Chicago: University of Chicago Press [Spanish translation: $E l$ marco conceptual de la psicología. Madrid: Debate, 1989].

Dewey, J. (1896). The reflex arc concept in psychology, Psychological Review, 3, 357-370 [Spanish translation: El concepto de arco reflejo en psicología. In J.M. Gondra (Ed.), La psicología moderna (pp. 197-207). Bilbao: Desclée de Brouwer, 1982].

Fuentes, J.B. (1989). ¿Funciona, de hecho, la psicología empírica como una fenomenología del comportamiento? Introducción a E. Brunswik. El marco conceptual de la psicología (pp. 777). Madrid: Debate.

Fuentes, J.B., \& Quiroga, E. (2001). Reformulación de las relaciones entre los condicionamientos operante y respondiente: El sentido de la crítica de J. Dewey al concepto de arco reflejo. Revista de Historia de la Psicología, 22, 327-333.

Koffka, K. (1935): Principles of Gestalt psychology. New York: Harcourt Brace. [Spanish translation: Principios de Psicología de la Forma. Buenos Aires: Paidos, 1953].

Pavlov, I.P. (1903/1982). Psicología y psicopatología experimentales en los animales. In I. P. Pavlov (Aut.), La actividad nerviosa superior. Obras escogidas (pp. 109-122). Barcelona: Fontanella.

Pavlov, I.P. (1917/1982). La fisiología y la psicología en el estudio de la actividad nerviosa superior de los animales. In I. P. Pavlov (Aut.), La actividad nerviosa superior. Obras escogidas (pp. 287-300). Barcelona: Fontanella.

Pavlov, I.P. (1933/1982). Sobre la posibilidad de fusión de lo subjetivo y lo objetivo. In I. P. Pavlov (Aut.), La actividad nerviosa superior. Obras escogidas (pp. 333-334). Barcelona: Fontanella.

Pavlov, I.P. (1934/1982). El reflejo condicionado. In I. P. Pavlov (Aut.), La actividad nerviosa superior. Obras escogidas (pp. 180-198). Barcelona: Fontanella.

Tolman, E. C., \& Brunswik, E. (1935). The organism and the causal texture of the environment. Psychological Review, XVII, 43-77.

Received May 20, 2003

Revision received September 29, 2003

Accepted October 3, 2003 